\title{
Information systems research in the media industry
}

\author{
Artur Lugmayr ${ }^{1}$
}

Received: 8 December 2016 / Accepted: 8 December 2016 / Published online: 26 December 2016

(C) Institute of Applied Informatics at University of Leipzig 2016

This special issue follows up past issues as e.g. (Alt and Zimmermann 2014) in contributing to business models in particular industry domains. However, this special issue is centred on media industries. The special issue is composed of 3 contributions. One of them can be seen as an introduction of the research field information systems in electronic media industry. The other two contributions focus on two different aspects: the first illustrates today's major problem of traditional media industries and deals with how new technologies can be utilized to transform these towards fully digital media houses; the second emphasizes the importance of applying concepts typically applied in media industry in the domain of information systems research.

With this special issue, we would like to stress the importance of putting more emphasis on media industry research within the agenda of information systems research. Even though media industry's IT spending is rather large, it is often neglected in information systems research. Research in media industry is rather cross-disciplinary, and the main research fields contributing to the advancement of the field are media and communication studies, media management, content production, the arts and creative fields, media and multimedia technologies, and business research. With the emergence of new technologies, such as the Internet, and digitalization of content business, many traditional media companies face the challenge of how to transform into fully digital media houses. Online service businesses disrupt old business strategies, and let new

Artur Lugmayr

artur.lugmayr@artur-lugmayr.com

1 Visualisation and Interactive Media (VisLab), Curtin University, Perth, Western Australia, Australia digital service companies emerge. To cope with these challenges, this special issue discusses the benefits for media industry that information system research provides. Additionally it discusses how information systems research can benefit from research conducted for media industries.

The contribution by Tassilo Pellegrini entitled "Semantic metadata in the publishing industry - technological achievements and economic implications" discusses the issues that the publishing industry has faced through the transformation towards digital. The contribution highlights the importance of metadata (Lugmayr et al. 2004) in the industry. It emphasizes the important role of data management practices on an organisational level, by introducing semantics and linked data to the example of publishing industries.

While Tassilo Pellegrini's contribution centred around the issue of how traditional media industries can cope with the transformation towards digital media houses, the contribution by Kai Huotari and Juho Hamari is an illustration of how concepts from media industries can be applied to information systems research. Kai Huotari and Juho Hamari's contribution "A definition for gamification: anchoring gamification in the service marketing literature" discusses the important aspect of gamification for service marketing. This contribution is an excellent example of how media research can contribute to the larger body of knowledge in information systems.

Both of the previously mentioned contributions focus on concentrated aspects in the media industry. The third contribution to the special issue by Artur Lugmayr and Johanna Grueblbauer offers a more holistic view on media industry and information systems research. The contribution entitled "Information Technology in the Media Industry" investigates the importance of media industry research in information systems. The large body of AIS contributed research work has been examined to identify how current IS research contributed to issues predominantly existing in media industry. The contribution also gives 
a clear definition of information systems research in media industries.

Readers who are interested in investigating the topic matter further, are very strongly advised to take a look at the following resources:

- Association of Information Systems (AIS) Special Interest Group on Electronic Media (AIS SIG eMedia) (SIGeMedia, n.d.);

- The email list of the AIS SIG eMedia, which is also available to members outside of the SIG and can be found on the SIG website as well (SIG-eMedia n.d.);

- Establishment of a publication series, and journal series entitled "Information Systems and Management in eMedia Industries" under open access (see (Lugmayr, A. (Ed.) 2013a) and (Lugmayr, A. (Ed.) 2013b));

- Past research activities in the context as e.g. the development of a European research agenda (Lugmayr 2013c), workshops (see i.e. (Lugmayr and Stojmenova 2013a), (Lugmayr and Stojmenova 2013b), and (Stojmenova and Lugmayr, A. (Eds.) 2013)) and some selected scientific works (Lugmayr 2013d), (Stockleben and Lugmayr 2013) and (Scheib 2017);

We hope the reader will enjoy this contribution, and that it will be an invitation to work in the fascinating domain of applying information systems research in the industry segment of media industries.

\section{References}

Alt, R., \& Zimmermann, H.-D. (2014). Editorial 24/4: electronic markets and business models. Electronic Markets, 24, 231-234.

Lugmayr, A., (2013c). Issues \& Approach in Defining a European Research Agenda on Information Systems and Management in Creative eMedia Industries, in: Stojmenova, E., Lugmayr, A. (Eds.), Proceedings 1st Workshop Defining European Research Agenda Information Systems Management eMedia Industries (inconjuction eBled, Bled, Slovenia), International Series Information Systems Management Creative eMedia.lugymedia
Inc., International Ambient Media Organization (AMEA), Bled, Slovenia, pp. 17-25.

Lugmayr, A. (Ed.), 2013b. International Series on Information Systems and Management in Creative eMedia (Open Access). International Ambient Media Association (AMEA) Ry, Tampere, Finland.

Lugmayr, A., (2013c). Issues \& approach in defining a European Research Agenda on information systems and management in creative eMedia industries. In E. Stojmenova, A. Lugmayr (Eds.), Proceedings 1st Workshop Defining European Research Agenda Information Systems Management eMedia Industries (inconjuction eBled, Bled, Slovenia), International Series Information Systems Management Creative eMedia (pp. 17-25). Bled: lugymedia Inc., International Ambient Media Organization (AMEA).

Lugmayr, A., (2013d). Research themes in informaiton systems \& management research in creative emedia industries, in: Lugmayr, A., Franssila, H., Karkkainen, H., Paavilainen, J. (Eds.), Proceedings 17th International Academic MindTrek Conference. Association for Computer Machinery (ACM), Tampere, Finland.

Lugmayr, A., \& Stojmenova, E., (2013a). 1st Workshop on Defining a European Research Agenda on Management Information Systems in eMedia Industries - information Management \& Systems in Media, Entertainment, Art, Education, and Culture Industries (MIS-Agenda@eMedia), in: Wigand, D.L., Carlsson, C., Clarke, R., Pucihar, A., B or štnar, M.K. (Eds.), 26thBledeConferenceChallenges ImpactsIndividuals, Organizations Society. University of Maribor.

Lugmayr, A., \& Stojmenova, E. (2013b). 1st Workshop on Defining a European Research Agenda on Management Information Systems in eMedia Industries - Information Management \& Systems in Media, Entertainment, Art, Education, Entertainment, and Culture Industries (MIS-AGENDA@eMedia). Bled, Slovenia.

Lugmayr, A., Niiranen, S., \& Kalli, S. (2004). Digital interactive TV and metadata - future broadcast multimedia. New York: SpringerVerlag.

Scheib, C. (2017). Business Information Management in Media Industries Accross Various Business Activities.

Stockleben, B., \& Lugmayr, A. (2013). Issues and topics to consider for information management research in eMedia industries. In: E.S. Asensio, A. Lugmayr, B. Stockleben, T. Risse, B. Pogorelc (Eds.), Proceedings 6th International Workshop Semantic Ambient Media Experiences (SAME2013), International Series Information Systems Management Creative eMedia (pp. 42-46). Bled: AMEA.

Stojmenova, E. \& Lugmayr, A. (Eds.), (2013). Proceedings of the 1st Workshop on Defining a European Research Agenda on Information Systems and Management in eMedia Industries, International Series on Information Systems and Management in Creative eMedia. lugYmedia Inc., International Ambient Media Organization (AMEA), Tampere, Finland. 